\title{
Article \\ The Prominent Role of Translation Factors, Heat shock Protein 90-2 and an ABC Transporter Associated to the Resistance of a Maize Line against Sugarcane Mosaic Virus
}

\author{
Gustavo Rodríguez-Gómez ${ }^{1}$, Pablo Vargas-Mejía ${ }^{1}$ and Laura Silva-Rosales ${ }^{1, *}$ \\ 1 Laboratorio de Interacciones Planta-Virus, Departamento de Ingeniería Genética, Centro de Investigaciones \\ y de Estudios Avanzados, Unidad Irapuato Libramiento Norte Carretera Irapuato-León, Irapuato, Gto. \\ 36824, Mexico; gustavo.rodriguez@cinvestav.mx (G.R.G); pablo.vargas@cinvestav.mx (P.V.M.) \\ * Correspondence: laura.silvar@cinvestav.mx
}

\begin{abstract}
The mosaic disease in maize is caused by Sugarcane mosaic virus (SCMV), a member of the Potyviridae family. The best strategy to cope with viral infections is the use of disease-resistant maize lines. To better understand the resistance response to SCMV, we analyzed differentially expressed genes among a resistant line (CI-RL1), a susceptible line (B73), and the F1 progeny from a cross between both lines using RNA-Seq data. We also analyzed transcript expression pattern clustering to allocate previously reported resistance candidate genes. GO enrichment analysis of biological processes highlighted a strong regulation in ROS detoxification in both the susceptible and resistant lines. The enrichment of cellular components led to the identification of an integral component of the plasma membrane in the RL line. Transcript expression patterns provide evidence of the importance of host translation in virus response, showing the diverse and complex behavior of $e I F 4 E$ homologs and the presence of eleven $e E F 1 \alpha$ factors in maize. In addition, we identified two genes putatively implied in long-distance movement: ZmPiezo and ZmPVIP1. Finally, we propose an $\mathrm{ABC}$ transporter to be associated with viral resistance.
\end{abstract}

Keywords: SCMV; Potyvirus; Resistance; RNA-Seq; Maize; eIF; eEF

\section{Introduction}

Maize is one of the most important food and staple crops around the world. However, like other important crops, it is affected by a variety of pathogens. The mosaic disease in maize is caused by Sugarcane mosaic virus (SCMV), a member of the Potyviridae family. Disease symptoms include leaf mosaics, chlorosis, and stunting, depending on the infected maize variety. SCMV has been shown to establish synergistic infections with Machlomovirus (Tombusviridae family), causing Maize lethal necrosis (MLN) [1], with devastating effects (with losses of up to 90\%) in maize cultivars in China and Africa [2,3]. The best strategy to cope with such viral infections is the use of resistant maize lines. However, the mechanism underlying maize resistance remains poorly understood.

The defense against plant pathogens relies on the innate immunity of each plant cell and on the systemic signals produced at the infected sites [4-7]. Plant innate immunity is activated after the recognition of microbial- or pathogen-associated molecular patterns (PAMPs and MAMPs) by transmembrane recognition receptors (PRRs) [6,7]. PAMPs-triggered immunity (PTI) is a general and non-specific defensive response, characterized by the activation of early responses such as production of reactive oxygen species (ROS), signaling cascade inducers (i.e., mitogen-activated protein kinases; MAPKs), and the accumulation of callose [6,8]. Pathogens can synthesize molecules that interfere with the activation of the PTI, which are called effectors [9]. If plants possess resistant (R) genes, proteins with nucleotide-binding sites (NBS), and leucine-rich repeats (LRR) domains [10], they can specifically recognize the effector, resulting in Effector-triggered immunity (ETI) [7]. ETI leads to the activation of ROS, MAPKs, and phytohormone signaling routes [11- 
14]. In addition, ETI also activates the production of defense proteins associated with cell death in a process called the hypersensitive response (HR) $[15,16]$.

Another defense mechanism against viral infection is RNA silencing $[17,18]$. The mechanism is triggered by the detection of double-stranded RNA (dsRNA). The dsRNA is cleaved by Dicer-like proteins (DCL) into small interfering 21-25 ribonucleotides (sRNAs). One of the dsRNA strands is loaded in the RNA-induced silencing complex (RISC) by the Argonaute (AGO) proteins. The RISC uses the sRNA as a guide to direct RNA degradation [17].

Another set of genes playing a role in resistance are the susceptibility factors. These are recessive genes playing a role in the virus infection cycle [19]. When one of these factors have mutations, the host-pathogen interaction is affected, with a concomitant resistance phenotype in the host. Most of the resistant genes to potyvirus identified in important crop plants correspond to alleles of the eukaryotic initiation factor 4E (eIF4E) [20].

Susceptibility factors, other than eIF4E, include those associated with long-distance virus movement. One of them is PVIP1 (Potyvirus VPg interacting protein) [21]. Mutants in Arabidopsis thaliana (L.) Heynh. that do not allow such interactions presented restriction of the long-distance movement of the virus, thus granting resistance to the viral infection.

The resistance to SCMV in maize has been assigned to the presence of the Scmv1 and Scmv2 loci in chromosomes 3 and 6, respectively. Both loci have been further explored, and the candidate genes were isolated. Scmv1 is an atypical thioredoxin $\mathrm{h}(\operatorname{Trx} \mathrm{h})$ [22], while Scmv2 is an Auxin-binding protein (ABP). Thioredoxins have a WC(G/P)PC motif, with two active-site cysteines (C) that participate in disulfide bridges reduction [22-24]. An atypical $Z m \operatorname{Trx} h$ has those two $C$ substituted with asparagine $(N)$ and serine $(S)$, yielding a WNQPS motif. ZmTrx h possesses 67 homologs in the maize genome, of which 32 are non-redundant without the WNPQS motif. It has been proposed that the Trx hinduced resistance was due to a higher gene expression, as it was also detected in susceptible plants. A mutation in the promoter region of $\mathrm{ZmTrx} h$ is responsible for the increase in transcript accumulation, but the mechanism related to resistance remains unclear [22].

To better understand the resistance response to SCMV, we analyzed differentially expressed genes between a resistant line (CI-RL1), a susceptible line (B73) and the F1 progeny derived from both lines. We carried out two main approaches with transcriptomic analyses: the first involved identification of the DEGs in the functional enrichment network of cellular components (CC), while the second involved transcript expression pattern clustering to allocate previously reported candidate genes. We observed, through GO enrichment analysis of biological processes (BP), a strong enrichment of ROS detoxification related genes. Furthermore, the enrichment of CC led to the identification of an ABC transporter. Transcript expression patterns provided evidence for the diverse (and, hence, complex) behavior of the eIF4E homologs in maize-particularly for the eEF1 $\alpha$ factors. Expression pattern analysis also allowed for the identification of two genes implied in long-distance movement: ZmPiezo and ZmPVIP1.

\section{Results}

\subsection{Symptom Development in Susceptible Line, Resistant Line, and F1 Progeny}

Six plants of each line (B73, CI-RL1, and F1 cross progeny) were grown in a greenhouse until they reached the third-leaf developmental stage. Then, all of their leaves were mechanically inoculated with SCMV-Ver1 isolate (EU091075.1). The plants were observed daily for the presence of mosaic symptoms. The susceptible B73 plants showed early mosaic symptoms in the ligule zone, as soon as $7 \mathrm{dpi}$. In contrast, CI-RL1 (RL) and F1 progeny (F1) plants were symptom-delayed, compared to B73 plants. RL 1 plants were almost identical to the uninfected controls, without any symptoms or developmental alterations. The F1 plants showed only a slight mosaic near the central vein and were like healthy plants (Figure 1A) with a slight mosaic in the youngest leaves. Chlorosis was observed in B73 plants, with a strong mosaic in the first- and second-youngest leaves, while a slight mosaic 
was observed in the basal region for the F1 leaves (Figure 1B). The F1 plants appeared to be taller than the other two lines, but this could be attributed to the heterosis effect.

\subsection{RNA-Seq of Virus-Infected Maize Plants}

To analyze the global host response to SCMV infection, we obtained the transcriptomes of B73, F1, and RL maize lines at $17 \mathrm{dpi}$, with their respective mock-inoculated control set and in duplicates. Therefore, a total of 12 libraries were pair-end sequenced (PE 2 $x$ 100), each one producing at least 20 million reads.

\subsection{Gene Expression Profiling is More Similar Between Susceptible B73 and Symptom-delayed F1, compared to RL $l$}

After the differential expression analysis, genes with $p$-value $\leq 0.01$ were considered as differentially expressed genes (DEGs). We found a total of 1959 DEGs in all maize lines and, of those, 924 corresponded to B73, 633 to F1, and 583 to RL. For B73, we found 496 upregulated and 459 down-regulated DEGs; 428 were up- and 206 down-regulated for F1; and 289 were up- and 304 down-regulated in the resistant CI-RL1 line. Only eight genes were differentially expressed in all three lines, corresponding to the $0.4 \%$ of the total DEGs (Figure 2A). The susceptible B73 line and the symptom-delayed F1 line were observed to be the most similar, sharing $5.7 \%$ and $3.2 \%$ of the up- and down-regulated genes (65 and 29 DEGs), respectively (Figures $2 \mathrm{~B}$ and $\mathrm{C}$ ). The resistant RL line shared only $1.1 \%$ and $2.3 \%$ of the total DEGs with F1 and B73, respectively. This feature-that is, fewer genes differentially expressed in the resistant line-is consistent with other transcriptomes when comparing resistant and susceptible lines. There are differences in the total (Figure 2A) and the addition of the up- down-regulated DEGs (Figures $2 \mathrm{~B}$ and $2 \mathrm{C}$ ) for each line. This is due to the different number of transcripts considered for each gene, behaving differently in each line, i.e., transcripts of the same gene being up-or down-regulated. Thus, the same gene will appear in both $2 \mathrm{~B}$ and $2 \mathrm{C}$ diagrams and, when added, the total number will be higher than in the $2 \mathrm{~A}$ diagram.

A

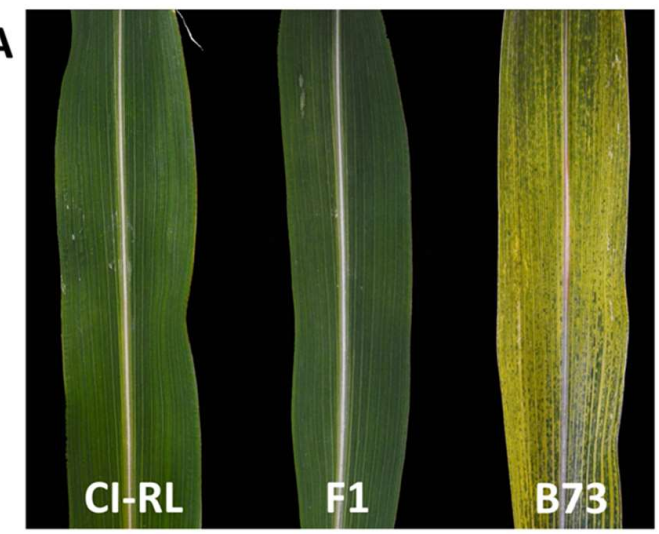

B

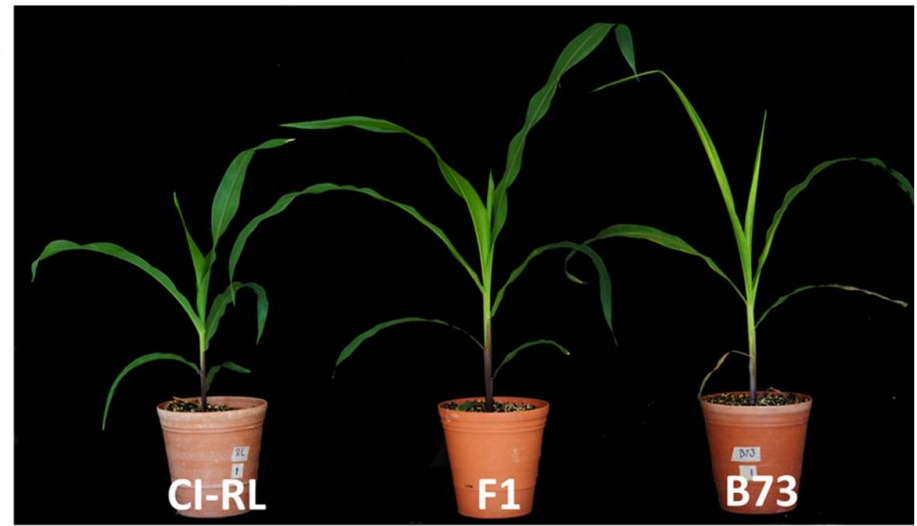

Figure 1. Comparison of symptom development between three maize lines with different responses to SCMV infection: A B73 susceptible-symptomatic line showing chlorotic mosaics, CI-RL (resistant) and F1 (delayed symptoms) do not show evident symptoms in the phenological equivalent leaves; B SCMV-infected plants with chlorosis in the first and second young leaves of the B73 plants, while a slight discoloration appears in the basal region of the second-and third-youngest leaves of the F1 plant. Chlorosis was not observed in planta in the RL line. The increased size of the F1 plant could be attributed to the heterosis effect. 

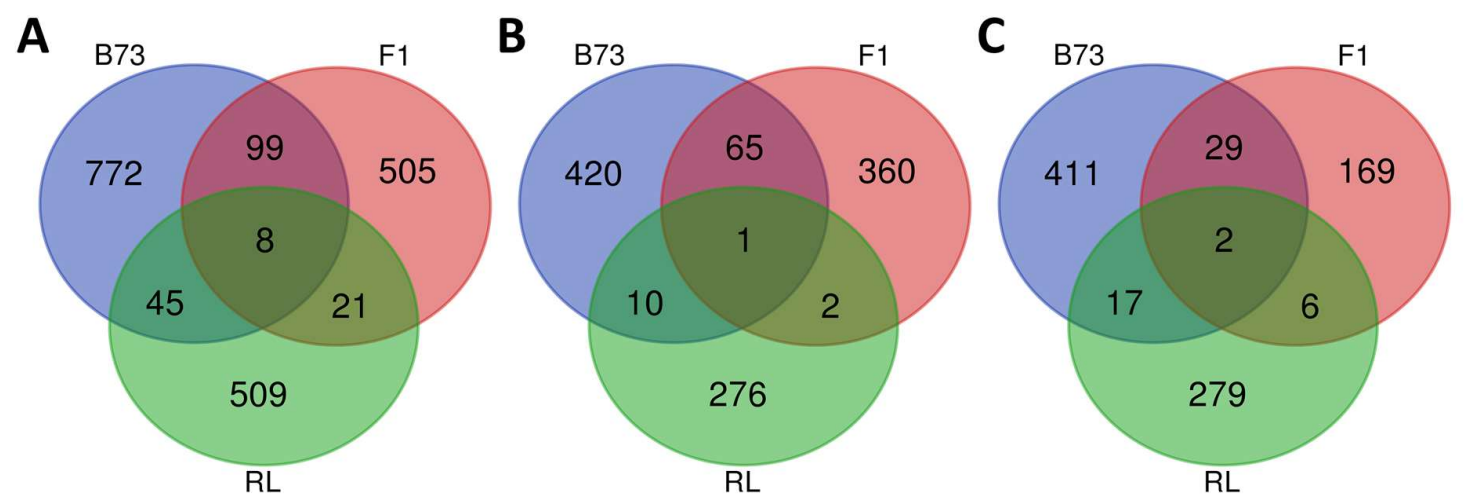

Figure 2. Differentially expressed genes showing B73 (susceptible-blue), F1 (delayed-symptoms-red), and RL (resistantgreen) line DEGs in Venn diagrams: (A) All differentially expressed genes (p-value $\leq 0.01)$; (B) Up-regulated genes; and (C) Down-regulated genes.

2.4. Membrane-Associated Cellular Components are Up- and Down-Regulated After SCMV Infection

To better understand the transcriptomic response of our three different maize lines to SCMV, we performed a functional enrichment analysis. A salient feature of the cellular component (CC) analysis enrichment was up-regulation of genes for the integral component of the plasma membrane in the RL line (Figure 3A). For B73 + F1, nine cellular processes were up-regulated: nuclear outer membrane-endoplasmic reticulum membrane network, cytosol, endoplasmic reticulum membrane, cytosolic ribosome, ribosomal subunit, proteasome complex, intracellular membrane-bounded organelle, non-membranebound organelle, and ribonucleoprotein complex. Five CCs were up regulated for B73+F1+RL: integral component of membrane, intrinsic component of plasma membrane, intrinsic component of membrane, cytoplasm, and intercellular organelle. Three CCs were up-regulated for B73+RL: microtubule, membrane-bound organelle, and nucleus. Most of the CCs were related to the network. On the other hand, nine down-regulated CC genes were found to be enriched only for B73 (Figure 3B), where four of them were connected and four did not have connections. Three down-regulated CCs were enriched for B73+F1+RL: integral components of membrane, intrinsic components of membrane, and membrane. They were connected only with intracellular anatomical structure for B73+RL (Figure 3B). 


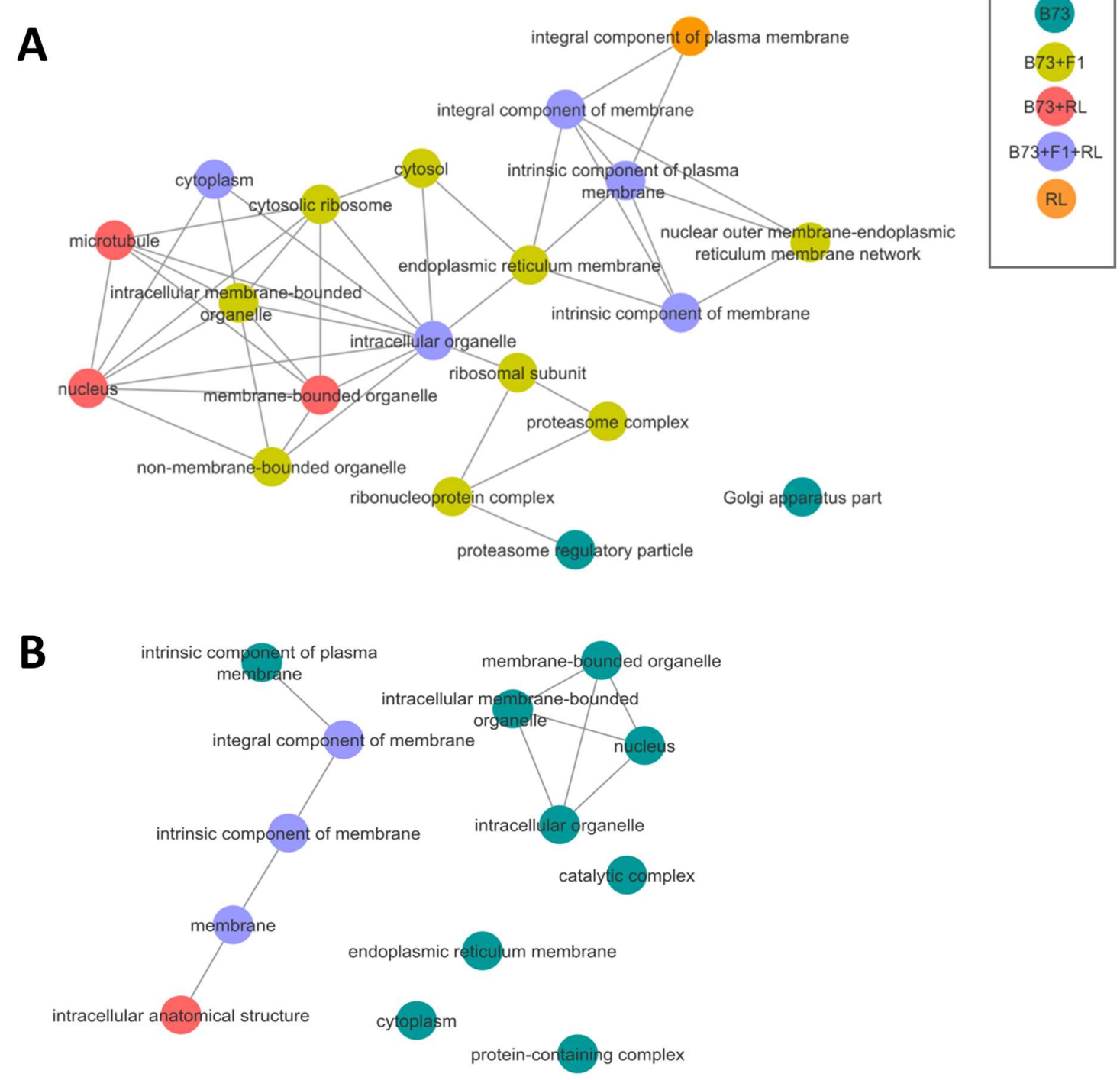

Figure 3. Functional enrichment network of cellular components (CC) of DEGs for lines B73 (susceptible), RL (resistant), and F1 (delayed symptoms): (A) Enrichment network of up-regulated genes; (B) Enrichment network of down-regulated genes. Nodes represent an enriched CC ontology term and edges represent the relationships between these ontologies. CC shared terms correspond to: B73 alone (dark green); between B73 and F1 (light green); between B73 and RL (red); shared between all lines (purple); and those corresponding to RL alone (orange).

\subsection{Detoxification, Membrane Fusion, and Chaperone-mediated Folding are Up-and Down-} regulated in $B 73$ and $R L$ lines.

Four biological processes (BPs) in the enrichment analysis were most up-regulated for B73 and F1 lines: translation, rRNA metabolic process, ribosome biogenesis, and ribonucleoprotein complex sub-unit organization. Four processes were also up-regulated for only B73: membrane fusion, hydrogen peroxide catabolic processes, chaperone-mediated protein folding, and cellular oxidant detoxification (Figure 4A). Fewer up-regulated genes in the RL line were related to protein metabolism and protein activation (regulation of protein metabolic process, regulation of phosphorous metabolic process, regulation of molecular function, protein-containing complex sub-unit organization, phosphorylation, and regulation of protein metabolic process). 
Down-regulation in BPs was observed in the resistant line (RL) for translation elongation, and that in translation initiation was observed for B73 (Figure 4B). Regulation of hydrolase activity seemed to be equally down-regulated for both B73 and RL lines. The most striking down-regulation of BPs was in chaperone-mediated protein folding for B73 and cellular oxidant detoxification for RL.

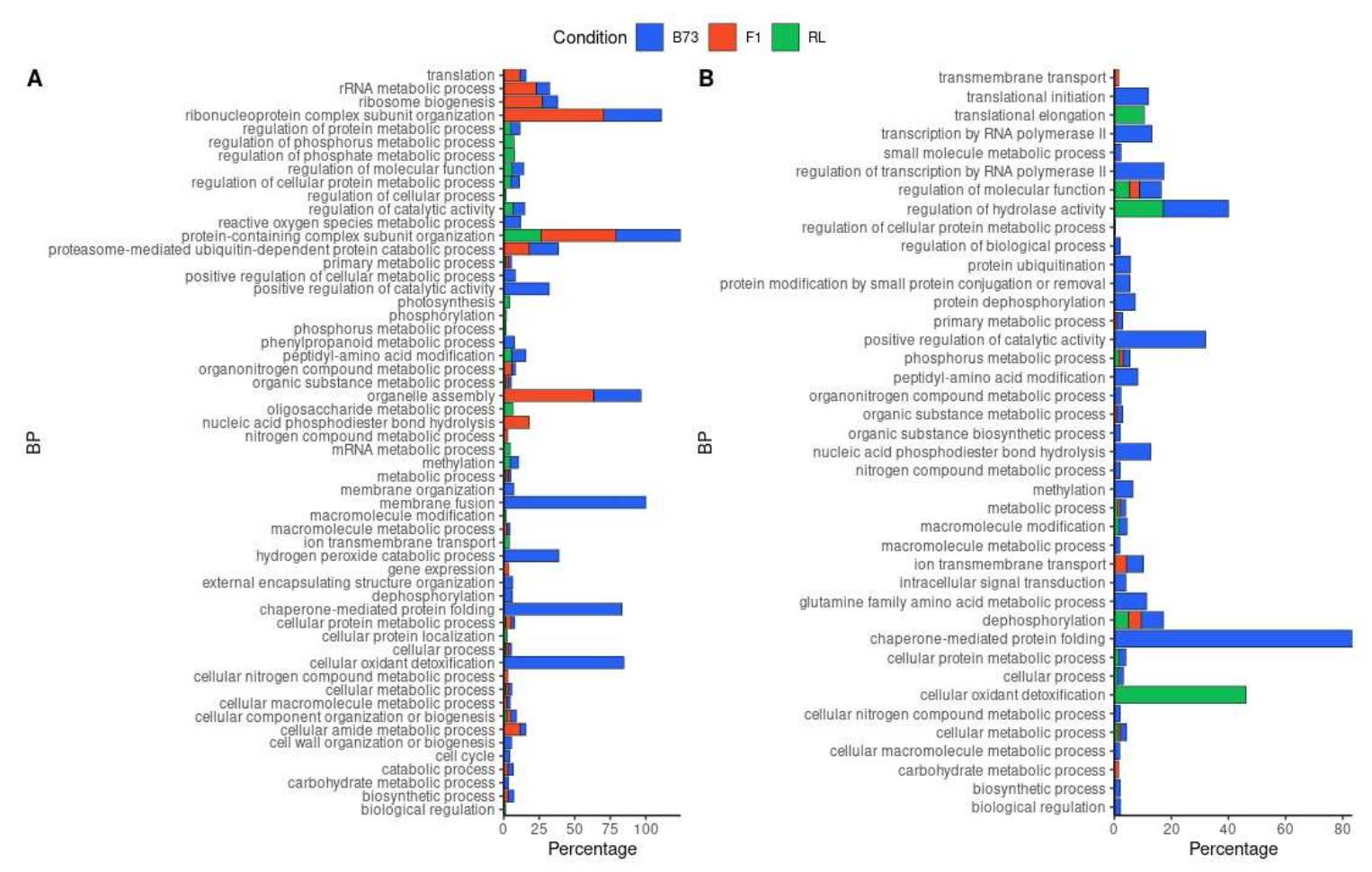

Figure 4. Bar plot of functionally up-regulated (A) and down-regulated (B) genes of enriched Biological Processes (BPs) for B73 (susceptible, in blue), F1 (delayed-symptoms, in red), and RL (resistant, in green) lines. (A) X-axis represents the percentage of background annotated genes at each BP therm.

\subsection{Resistance Candidate Genes: Mostly present in F1 and Down-regulated in B73.}

We looked for previously reported resistance candidate genes in the literature within our set of enriched BPs. Then, we selected some genes according to their up- or downregulated expression levels. In Table 1, we present 19 up- and 24 down-regulated selected candidate DEGs. The first set corresponds to eight down-regulated BPs: translation elongation, cellular detoxification, biological regulation chloroplast, response to external stimuli, regulation of gene expression, carbohydrate metabolic process, and digoxygenase activity. The second set (up-regulated) are within four enriched BP terms: translation, RNA metabolic process, gene silencing by miRNA, and response to stress. Within the set of 24 down-regulated genes, 16 are for the B73 line, with $\log _{2}$ FC values ranging from -1 to -6 (for Argonaute $1 \mathrm{~b}$ and Heat shock $70 \mathrm{kDa}$ protein 17, respectively), whereas only four were down-regulated for RL (Translation elongation factor EF1A, Elongation factor $1 \alpha$, Thioredoxin superfamily protein, and jasmonate-regulated gene 21). For the up-regulated set of genes, 12 were associated to the F1 line, 6 to B73, and 4 to RL. Translation was the mostly represented biological process, with five genes: elongation factor 2 and its sub-unit $\beta$, elongation factor $1 \alpha$, translation initiation factor $4 \mathrm{G}$, and translation initiation factor 3 sub-unit M. GO software sorts the genes in hierarchical groups. Therefore, a BP can encompass another BP explaining why the same gene can be found in just one or shared between two or more GO terms. This is why Thioredoxin superfamily protein is in two GO hierarchical terms, the GO:0065007-biological regulation and the GO:1990748-cellular detoxification. Meanwhile, another thioredoxin annotated as Thioredoxin-like 
4/Thioredoxin-like protein CXXS1 is only found in GO:0065007-biological regulation (Table 1) reflecting annotation and enrichment of GO differences. We have no evidence of the presence of ZmTrx $h$ within this group. Both argonaut1 and argonaut1b, were found in the GO:0035195-gene silencing but only argonaute1b found in GO:0065007- biological regulation. None of them were associated with resistance to SCMV.

\subsection{Most of the Candidate Genes Have Higher Basal Expresion in RL}

Analysis of the DEGs highlighted their possible role in SCMV resistance; for instance, eEFs, dicer-like, and argonaute were detected. However, this approach may have excluded recessive resistance genes or susceptibility factors with unchanged expression levels. In order to detect these types of genes, we grouped transcripts into patterns. Five expression pattern clusters were generated, showing basal expression levels of genes for each of the three evaluated lines. The pattern clusters were then grouped according to the basal level of expression for the RL line, thus resulting in groups 1 and 2 (Figure 5A). Group 1 had a high basal expression level for RL and included clusters C0, C1, and C2. Group 2 had a low level of basal expression for RL and contained clusters C3 and C4. Considering previous attempts to understand the resistance of maize to SCMV, a list of 57 genes involved in potyvirus resistance were extracted from bibliographic data [25,26]. We then searched for the list of 57 candidate genes in all the clusters and found 43 . Of these, there were 13 in group 1: two of them were associated with long-distance movement (PVIP, and ZmPiezo), three with cell-to-cell movement (BG3, Pcap1, and Myosin), and seven with the initiation or enhancement of viral replication (EXA1, LSM1, PpDDXL, IRE1A, RIM1, Chl-PGK, and RISP). Additionally, one of the detected genes (SAMS1) does not partipate in the virus infection cycle. Only three genes were found exclusively in group 2 (IRE1B; PDLP in C4; and PBAP8 in C3); see Figure 5B. The remaining 27 candidates were found in cluster combinations, as they had high and low levels of basal expression for RL (Figure 5B). This grouping helped us to understand the significant/relevant differences in transcript expression between the resistant line (RL) and the susceptible line (B73).

eIF4E was also observed in the expression pattern clusters, due to its importance in the potyvirus infectious cycle and its role as a susceptibility factor. Two candidate genes were identified in the maize genome: the first was annotated as eif6, and the second corresponded to the gene model GRMZM2G113096 (with no annotation and termed ZmeIF4E). The expression of eif6 had a low basal level in the F1 and RL lines, while eif7 and ZmeIF4E had a low basal level in the RL line. 


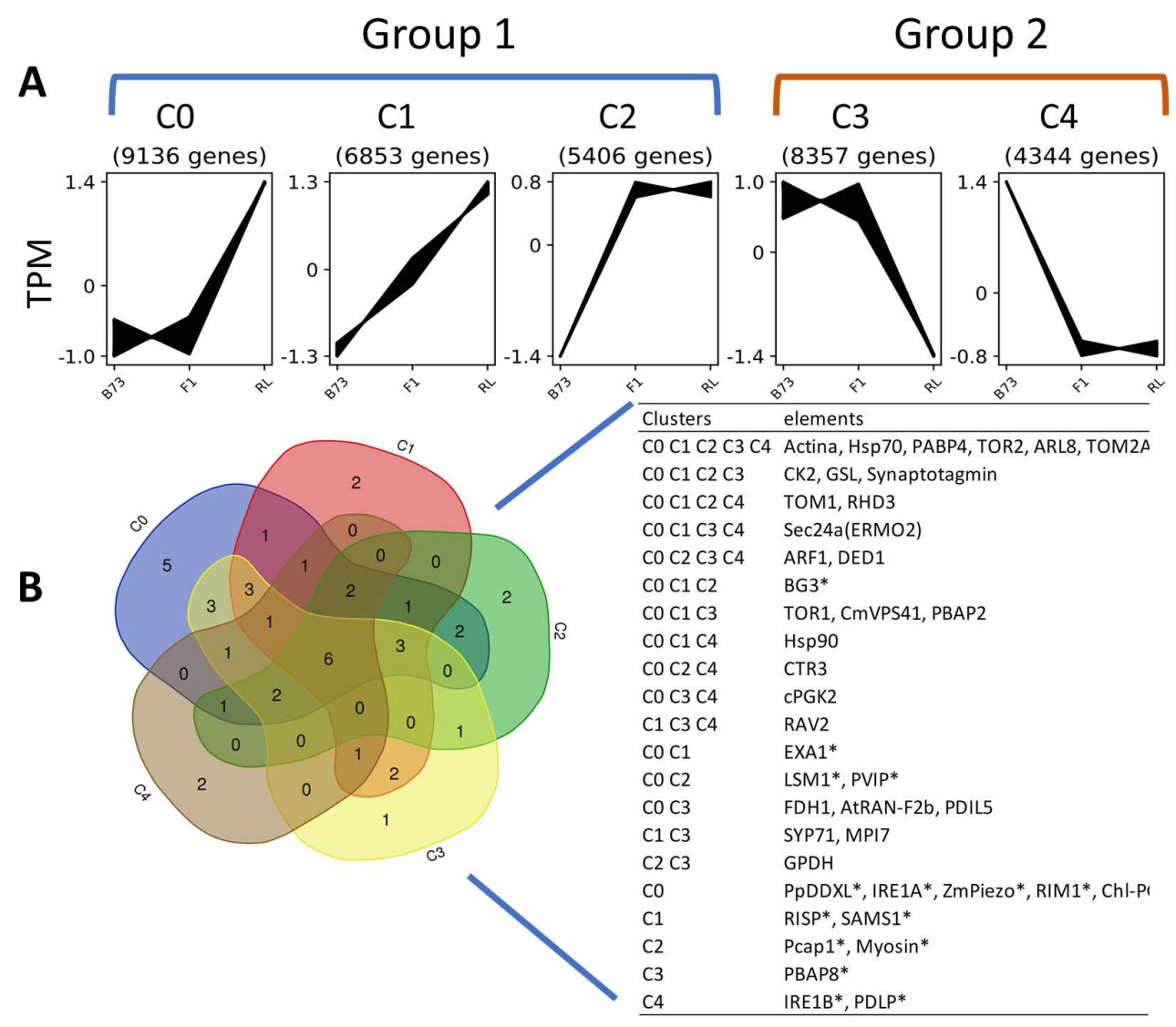

Figure 5. Transcript expression patterns in three maize lines: (A) Total clusters (C0 to C4) of transcript expression patterns (of non-DEGs) across the three infected susceptible (B73), resistant (RL), and the delayed-symptom F1 cross (B73 x RL) maize lines; and (B) Venn diagram of potyviral-resistance for 43 selected candidate genes present in one or more expression pattern cluster ( $\mathrm{C} 0$ to $\mathrm{C} 4$ ). ${ }^{*}$ Set of sixteen candidate genes found only in one cluster.

\subsection{Differences in Translation and Elongation Factor Coding Transcripts as the Possible Source of RL Resistance}

The interaction of the potyvirus VPg with the translation initiation factor eIF4E and/or its isoforms plays a central role in the virus infectious cycle [27]. A search in the maizeGDB database revealed that maize possesses six genes annotated as eukaryotic initiation factors (Table 2). In a prior search for homologs of the eIF4E from Arabidopsis in maize, the best candidate identified in the maize genome corresponded to the gene model GRMZM2G002616, annotated as eif6. As this factor was not found to be differentially expressed, its sequence was used to search within the expression pattern clusters. eif6 was found in the C4 cluster, with low levels of basal expression in both the RL line and the F1 progeny, while having high expression levels in B73. Additionally, two more eIFs were identified in cluster C3: one corresponding to the gene model GRMZM2G022019 and annotated as eif-7, and other corresponding to the gene model GRMZM2G113096 without annotation and assigned as ZmeIF4E.

In contrast, the eukaryotic elongation factors (EEFs), which also participate in protein production, presented interesting results. First, we found four differentially expressed gene models related to eEF1 $\alpha$ : GRMZM2G001327, annotated as elfa12; GRMZM2G154218m annotated as elfa3; GRMZM2G343543, annotated as elfa10; and GRMZM5G850607, not annotated and designated as ZmeIF4E. Furthermore, the behavior of each transcript was unique, and two were up-regulated: elfa10 in F1 progeny and the B73 line, while elfa3 only in the B73 line. In contrast, elfa12 and Zm-elfa showed downregulation only in the RL line. 
A further search in the MaizeGDB database for annotated eIFs and eEFs showed that maize possesses at least 6 genes annotated as eIFs and 11 genes annotated as eEFs (Supplementary Table 1). Additionally, as observed in the DEGs data and the transcript expression pattern clustering analysis, different gene models and different transcripts of the eIFs and eEFs were found, depending on the genetic background. Thus, the results of the response to SCMV could be the effect of the transcripts favored in each individual.

Table 1. Up- and down-regulated candidate genes from enriched BPs. Down- (in blue) and up-regulated (in red) genes, their GO terms, maize transcript IDs, fold-change expression levels $\left(\log _{2}\right)$, and associated annotation are presented. *The selected candidate from the integral component of plasma membrane enriched CC corresponding to an ABC transporter.

\begin{tabular}{|c|c|c|c|c|c|}
\hline \multirow{2}{*}{ Go term } & \multirow{2}{*}{ ID } & \multicolumn{3}{|c|}{$\log 2 \mathrm{FC}$} & \multirow{2}{*}{ Gene name } \\
\hline & & B73 & $\mathrm{F} 1$ & $\mathrm{RL}$ & \\
\hline \multirow{2}{*}{ Tranlational elongation } & Zm00001d025100 & & & -1 & Translation elongation factor EF1A ( $\mathrm{Zm}$-elfa) \\
\hline & Zm00001d037877 & & & -1 & Elongation factor 1-alpha (elfa12) \\
\hline Detoxification/Cellular detox & Zm00001d013348 & & & -6 & Thioredoxin superfamily protein \\
\hline \multirow{4}{*}{ Biological regulation } & Zm00001d026111 & -6 & & & argonaute1b \\
\hline & Zm00001d026368 & -1 & -1 & & Thioredoxin-like 4/Thioredoxin-like protein CXXS1 \\
\hline & Zm00001d017103 & -5 & & & Repressor of RNA polymerase III transcription \\
\hline & Zm00001d016410 & -1 & & & RNA-binding (RRM/RBD/RNP motifs) family protein \\
\hline Chloroplast & Zm00001d031953 & -5 & & & Glutathione S-transferase \\
\hline Response to external stimuly & Zm00001d052327 & & -5 & & Regulatory-associated protein of TOR 1 \\
\hline \multirow{13}{*}{ Regulation of gene expression } & Zm00001d005100 & -1 & & & RNA binding (RRM/RBD/RNP motifs) family protein \\
\hline & Zm00001d047574 & -5 & & & WRKY55, expressed \\
\hline & Zm00001d031953 & -5 & & & Glutathione S-transferase, $\mathrm{N}$-terminal domain containing protein Thioredoxin family \\
\hline & Zm00001d013307 & -5 & & & Probable WRKY transcription factor 74 \\
\hline & Zm00001d014793 & -1 & & & LRR-like kinase protein THICK TASSEL DWARF1 thick tassel dwarf1 \\
\hline & Zm00001d047209 & -1 & & & Calmodulin-binding transcription activator 2 \\
\hline & Zm00001d022088 & -1 & & & Agamous-like MADS-box protein AGL8 \\
\hline & Zm00001d051609 & -6 & & & Oxygen-regulated protein/Heat shock 70 kDa protein 17 \\
\hline & Zm00001d007949 & -5 & & & Agamous-like MADS-box protein AGL8 \\
\hline & Zm00001d023659 & -1 & & & Auxin response factor 2 \\
\hline & Zm00001d018178 & -1 & & & ABSCISIC ACID-INSENSITIVE 5-like protein 5 \\
\hline & Zm00001d018799 & & -1 & & $\begin{array}{l}\text { Non-specific serine/threonine protein kinase } \backslash \mathrm{CBL} \text {-interacting serine/threonine-pro- } \\
\text { tein kinase } 23\end{array}$ \\
\hline & Zm00001d015053 & & -1 & & Terpene synthase 2 \\
\hline Carbohydrate metabolic process & Zm00001d029313 & & -1 & & PLASMODESMATA CALLOSE-BINDING PROTEIN 5 \\
\hline Dioxygenase activity & Zm00001d012456 & & & -6 & jasmonate-regulated gene 21 \\
\hline
\end{tabular}

\begin{tabular}{|c|c|c|c|c|c|}
\hline \multirow{2}{*}{ Go term } & \multirow{2}{*}{ ID } & \multicolumn{3}{|c|}{ Log2FC } & \multirow{2}{*}{ Gene name } \\
\hline & & B73 & F1 & $\mathrm{RL}$ & \\
\hline \multirow{6}{*}{ Translation } & Zm00001d034776 & & 1.1 & & Elongation factor 2 \\
\hline & Zm00001d007179 & & 0.7 & & Eukaryotic translation initiation factor 2 subunit beta \\
\hline & Zm00001d037873 & 1 & & & Elongation factor 1-alpha (elfa3) \\
\hline & Zm00001d036904 & 0.9 & 0.7 & & Elongation factor 1-alpha (elfa10) \\
\hline & Zm00001d006573 & & 5.4 & & Eukaryotic translation initiation factor $4 \mathrm{G}$ \\
\hline & Zm00001d008757 & & & 5.9 & Eukaryotic translation initiation factor 3 subunit $\mathrm{M}$ \\
\hline \multirow{7}{*}{ RNA metabolic process } & Zm00001d042512 & 6.6 & 6.4 & & Ribonuclease 2 \\
\hline & Zm00001d023706 & 2.5 & & & thioredoxin $\mathrm{M} 1$ \\
\hline & Zm00001d049269 & & 5 & & RNA polymerase II C-terminal domain phosphatase-like 3 \\
\hline & Zm00001d044242 & 1.5 & 1.2 & & Transcription factor bHLH87 \\
\hline & Zm00001d051885 & & & 4.5 & ATG8-interacting protein 1 \\
\hline & Zm00001d040311 & & & 5.4 & 5'-3' exoribonuclease 3 \\
\hline & Zm00001d049680 & & 1.3 & & RNA-binding (RRM/RBD/RNP motifs) family protein \\
\hline \multirow{3}{*}{ Gene silencing by miRNA } & Zm00001d013796 & & 5.4 & & dicer-like 105 \\
\hline & Zm00001d040082 & 5.6 & & & DExH-box ATP-dependent RNA helicase DExH3 \\
\hline & Zm00001d011096 & & 0.9 & & argonaute1 \\
\hline \multirow{3}{*}{ Response to stress } & Zm00001d034768 & & 5.6 & & Glycine-rich RNA-binding protein RZ1A \\
\hline & Zm00001d051885 & & 0.7 & & ATG8-interacting protein 1 \\
\hline & Zm00001d031332 & & & 1.7 & Heat shock protein $90-2$ \\
\hline Integral to Plama Membrane* & Zm00001d008178 & & & 5.9 & ABC transporter B family member 21 \\
\hline
\end{tabular}

Table 2. Transcripts coding for translation/elongation factors found in clusters C0 to C4 (see Figure 5A). 


\begin{tabular}{|c|c|c|c|c|c|c|c|c|c|}
\hline \multicolumn{2}{|r|}{$\mathrm{CO}$} & \multicolumn{2}{|r|}{ C1 } & \multicolumn{2}{|r|}{$\mathrm{C} 2$} & \multicolumn{2}{|r|}{$\mathrm{C3}$} & \multicolumn{2}{|r|}{ C4 } \\
\hline Gene & Transcript ID & Gene & Transcript ID & Gene & Transcript ID & Gene & Transcript ID & Gene & Transcript ID \\
\hline elfa1 & Zm00001d009868_T004 & elfa1 & Zm00001d009868_T009 & elfa1 & Zm00001d009868_T003 & ZmelF4E & Zm00001d041973_T001 & eif-6 & Zm00001d041682_T001 \\
\hline elfa2 & Zm00001d037875_T005 & elfa2 & Zm00001d037875_T008 & elfa1 & Zm00001d009868_T012 & eif-7 & Zm00001d014065_T001 & elfa2 & Zm00001d037875_T006 \\
\hline elfa3 & Zm00001d037873_T011 & elfa3 & Zm00001d037873_T009 & elfa2 & Zm00001d037875_T003 & elfa1 & Zm00001d009868_T002 & elfa3 & Zm00001d037873_T001 \\
\hline elfa9 & Zm00001d046449_T004 & elfa10 & Zm00001d036904_T002 & elfa11 & Zm00001d037905_T003 & elfa12 & Zm00001d037877_T005 & elfa7 & Zm00001d009870_T013 \\
\hline elfa11 & Zm00001d037905_T002 & elfa10 & Zm00001d036904_T005 & elfa12 & Zm00001d037877_T008 & & & & \\
\hline
\end{tabular}

\section{Discussion}

Maize resistance to SCMV was reported more than 30 years ago [28]. However, two genes proposed for resistance-thioredoxin and nABP, corresponding to the loci Scmv1 and Scmv2, respectively-have recently been discovered. The selected line, CI-RL1, used in this work was identified in inbred evaluations for resistance to potyviruses at CIMMYT. We have previously shown that long-distance movement of SCMV is impaired in this line [29] and, therefore explored the possibility of potyvirus movement restriction involved in resistance, along with other 43 selected candidate genes. The latter were searched for in a transcript expression pattern clustering and/or in DEGs derived from their expression using RNAseq analysis.

\section{DEGs and GO Pinpoint Viral Replication Complexes, detoxification and chaperones}

As observed in planta, the presence and severity of symptoms were more intense in B73. Correspondingly, most of the differences in DEGs related to BPs were found in this maize line and were associated with the homeostatic equilibrium maintenance response, such as membrane fusion, peroxide hydrogen catabolic process, chaperone-mediated protein folding, and cellular oxidant detoxification (Figure 4A).

An increase in the membrane fusion $\mathrm{BP}$ is not surprising during potyviral infection. The formation of vesicles, such as viral replication complexes (VRCs), is an essential step in this interaction. Inside these vesicles, host cellular components are recruited and used for virus replication [30,31], along with dsRNA intermediaries, which have been suggested to subvert the RNA silencing-based defense mechanism [32-34]. Some potyviruses use the chloroplast and ER membranes $[34,35]$ to produce VRCs, with concomitant chlorophyll breakdown and leaf yellowing during severe chlorosis [36-38], which was also consistent with the GO term enrichment analysis (Figure 4A). In fact, this could also explain the increase in the number of genes involved in the hydrogen peroxide catabolic process and in cellular oxidant detoxification. The liberation of fragments or the content of some organelles could act as signals in the attack of a pathogen in the so-called damageassociated molecular pattern (DAMPs), generating a ROS burst response [39-42]. The over-accumulation of ROS species (also called oxidative stress) can damage and disrupt the function of cellular components [43,44]. Under normal physiological circumstances, the production and degradation of ROS species are in a steady state. Therefore, due to the constant production of ROS species in the cell, detoxification of the inner cell environment is necessary. This could explain the increased expression levels in RNA-seq data of genes involved in these processes (Figures $4 \mathrm{~A}$ and B). The cellular oxidant detoxification genes were up-regulated in the resistant CI-RL1 line. This up-regulation could be explained as an effective and transient defense response to prevent further cellular damage. Thus, this increase appears to be more a consequence of an early response to infection, rather than a strategy used for resistance.

Chaperones associated BP were also enriched. They participate in protein folding, enabling mis-folded or aggregated proteins to correctly fold [45,46], and are also involved in targeting proteins for degradation [47]. They are also activated in response to biotic and abiotic stresses. Chaperone-mediated protein folding was up- and down-regulated in the B73 line (Fig. 4B). Potyviruses recruit the heat-shock protein 70 (Hsp70) chaperone in the formation of replication complexes [48,49]. Another pair of Hsps-Hsp40 and Hsp90appear to be involved in viral infections [50-52]. We detected the up regulation of the Heat shock protein 90-2, only in the RL line (Table 1). Furthermore, we corroborated this 
increase in its expression level by qRT-PCR (Figure S4) suggesting its association with resistance.

Integral $A B C$ Transporter in Resistant Maize Line

The only component enriched for the resistant RL line belonged to the integral components of the cellular membrane. One candidate was especially noteworthy: the ATPbinding cassette $(A B C)$ transporter corresponding to the maize GRMZM2G085111 gene model (Table 1). ABC transporters move diverse structural unrelated components across the membrane and intervene in plant-pathogen interactions [53-56]. A wheat ABC transporter (lr34 gene) has been reported as a multi-disease resistance gene (MDR) against fungal pathogens [57,58]. Furthermore, maize plants expressing the Lr34 wheat gene showed resistance against two fungal pathogens [59]. The protein codified by the maize gene model GRMZM2G014282 has been identified as the best homolog to the Lr34 protein. The GCN-type ABC transporter from Lilium regale E.H.Wilson has been shown to be involved not only in the defense against fungi, but also against viruses [56]. This pathogen resistance is not due simply to the presence of the ABC transporter, as has been discussed by Sun et al. (2016) [56]: a more complicated network of genes, in which the transporter plays a pivotal role, appears to be involved. Overall, GRMZM2G085111 deserves further research.

\section{Genes Involved in Virus Replication Found in Cluster Pattern Analyses}

We found two candidates associated with virus replication (in group 2, Fig. 5): PolyA Binding protein 8 (PABP8) and inositol-requiring protein 1B (IRE1B). The PABPs bind to the 3'-end of mRNA. Three (PABP2, PABP4, and PABP8) out of eight isoforms in Arabidopsis showed increased protein and mRNA levels during TuMV infection, besides the interaction of PABP2 with the VPg and RdRp of TuMV. Also, in pabp2pabp4 and pabp2pabp8 double mutants, a reduction in TuMV mRNA levels was observed [60]. While homologs for the three AtPABPs were searched, only PABP8 was detected in Group 2 of C3 cluster (Table in Figure 5B), possibly due to its implication in virus accumulation.

IRE1B and IRE1A homologs in Arabidopsis are involved in the splicing of $b$ ZIP60 in the response to biotic and abiotic stresses, resulting in the activation of stress-related proteins. The ire1aire1b double mutant inoculated with TuMV showed delayed symptoms and low virus accumulation [61]. Thus, both IRE1 genes are necessary in Arabidopsis to confer resistance. In maize, IRE1A was found in group 1, while IRE1B was found in group 2. As IRE1A and IRE1B appears to act redundantly, they may not participate in RL resistance.

The candidate genes EXA1, LSM1, PpDDXL, IRE1A, RIM1, Chl-PGK, and RISP (Table S2), with high levels of basal expression in the RL line (group 1), play roles in viral replication and, therefore, were not considered to be associated with resistance.

\section{Cell-to-cell Movement-Related Genes}

Viruses move from cell to cell through plasmodesmata (PD), which regulate the size exclusion limit (SEL) through callose accumulation and the participation of viral movement proteins (MPs). Potyviruses, not having a specialized MP, rely on the CI, CP, HCPro, and VPg proteins for cell-to-cell movement, inducing the formation and movement of VRCs, via the cytoskeleton to and through the PD, in association with CI [62]. Therefore, proteins that modify or interact with the PD can be considered important candidates in cell-to-cell movement. We found candidates involved in cell-to-cell movement in group 1 (BG3, PCap1, and Myosin) and in group 2 (PDLP) (Table in Fig. 5B).

The first of the candidates in group 1, $\beta$-1,3-glucanase (BG3), was found in the three clusters of groups 1, in Fig 5B (C0, C1, and C2). BG3 encodes an enzyme that degrades callose deposited in the plasmodesmata [63]. Callose deposition and its degradation are controlled by two enzymes: callose synthase (CalS) and BG3, respectively. It has been 
suggested that callose deposition restricts the spread of pathogens [64-66]. It is tantalizing to speculate that the oxidative stress indicated by the RNAseq data could have led to callose accumulation in the PD [67]. This accumulation requires the activation of BG3 for its degradation; however, experimental support is required to confirm such an association.

The second candidate of group 1 in Fig. 5 was the plasma membrane-associated cation-binding protein 1 (PCap1). The expression level of PCap1 in Capsicum annum L. has been shown to be related to Potato virus $Y$ accumulation and cell-to-cell movement [68]. Furthermore, protoplasts with a pcap1 loss-of-function mutation in A. thaliana accumulated high levels of TuMV, discarding its participation in virus replication [69]. Additionally, the interaction between PCap1 and the P3N-PIPO from TuMV appears to be involved in the localization of the CI complex at PD [62,69]. As PCap1 favors a possible increase in cell-to-cell transport, its involvement in SCMV resistance appears unlikely.

The third candidate gene from group 1 was Myosin, a motor protein involved in a variety of mobility processes. As such, the silencing of Myosin XI-2 from Nicotiana benthamina Domin has been shown to inhibit the movement of the Tobacco mosaic virus (TMV), but not for Potato virus X (PVX) and Tomato bushy stunt virus (TBSV) [70]. Apparently, as plants possess different genes that code for myosin, viruses interact selectively with them. This Myosin homolog was found to have a high expression level in the RL line, which could favor SCMV accumulation and movement, thus not enhancing host resistance.

The only candidate in group 2 associated with cell-to-cell movement was a PD-located protein (PDLP) belonging to a family of type-I membrane proteins. These proteins travel along the secretory pathway to reach the plasma membrane inside the PD [71,72]. PDLPs interact with viral movement proteins (MPs), which are capable of assembling as tubules within the PD [71]. As potyviruses do not belong to the group of tubule-forming viruses, the role of PDLP in SCMV-infection remains uncertain.

\section{Long-distance Movement-Related Genes}

Regarding long-distance movement and the need to systemically infect the host, only two candidates were found in group 1 (Fig. 5): the Piezo and PVIP homologs. The ZmPiezo gene was chosen from the maize genome as an ortholog to the ESC1 gene from Arabidopsis. ESC1 codes for an ion channel, PIEZO, that responds to mechanical stimuli [73]. The esc1 mutant showed alteration in the long-distance movement of TuMV in A. thaliana [74]. The ESC1 gene, further called AtPiezo, was transcriptionally induced by viral infection [74]. The ZmPiezo gene was found to have a high level of basal expression in the RL line. Not know much about ZmPiezo in maize, so it is difficult to say whether its high level can be associated with SCMV resistance.

Considering the Potyviral VPg Interacting Protein 1 (PVIP1), the pvip1 loss-of-function mutant in $A$. thaliana inoculated with TuMV resulted in aberrant phenotypes and a lack of viral symptoms in planta [21]. This could be interpreted as the need for PVIP1 to ensure viral infection. Here, not only the RL line has this gene, but it was found in high basal levels (Figure S4) with possible SNPs.

The Presence of Diverse Transcripts for eIFs and eEFs Imply a Complex Regulation of Translation in SCMV Interaction with Maize Transcripts in Resistance

Of paramount importance in this work is the role that translation seems to be playing in RL resistance involving factors in its initiation step. This was not surprising as several of these initiation factors have been well documented during the last decades. However, little is now for the role of elongation factors in viral infection. Eukaryotic initiation factors (eIF), had negative regulation in BPs for the B73 line (Figure 4B). It appears that the expression level of eIF4E is important for the establishment and maintenance of viral infection. Plants that transiently overexpress a modified eIF4E showed an increase in resistance to Potato virus $Y$ and a decrease in the expression level of the host gene [75]. Inhibition of cap-dependent translation, by the interaction of the host eIF4E with $\mathrm{VPg}$, favors 
the use of Internal Ribosomal entry sites (IRES) in the 5' UTR region of some potyviruses [76]. Furthermore, different kinds of stresses appear to stimulate IRES translation [76,77] and down-regulate the production of new host proteins [78], thus influencing the eIFs. This would be the case for the stress caused by SCMV infection.

The ortholog for the eIF4E from Arabidopsis (AT4G18040) corresponds to the maize eif-6 transcript (Zm00001d041682), one of the six genes annotated as eukaryotic initiation factors in the maizeGDB database (Table S1). Transcript eif-6, within cluster C4 (Table 2 and Fig. 5A), has low basal expression levels in the resistant line (RL) and the late-symptom F1 line, implying its participation in the virus infection cycle. On the other hand, eif7 and ZmeIF4E had low basal expression levels only in the RL plant. The best match in the Arabidopsis genome for the eif-7 genes corresponds to the isoform of the eIF4E factor. The use of the eIF4E or its isoform depends completely on the interaction of the host and the infecting virus. Both eif-7 and ZmeIF4E can be newly described alleles in maize associated with resistance, for instance, restricting viral movement in the RL line, as has benn described for TEV in A. thaliana [79]. Both B73 and RL had different basal levels of ZmeIF4E (Table 2), suggesting either differences in their coding or in the promoter region for this gene.

The eukaryotic elongation factors (eEFs) are encoded by genes playing a central role in the elongation step of translation [80]. One of them-namely, eukaryotic elongation factor $1 \alpha(\mathrm{eEF} 1 \alpha)$-is a cytoplasmatic protein that delivers aminoacylated tRNAs to ribosomes during polypeptide elongation when bound to GTP [81,82], and play a role in nuclear export, proteolysis, and apoptosis [83]. In human viral infections, eEF1 $\alpha$ has been shown to interact with the Gag1 polyprotein of the Human immunodeficiency virus type 1 (HIV-1) [84], the 3'stem-loop region of the West Nile virus (WNV) [85], and with the nucleocapsid protein of the Paramyxovirus Respiratory Syncytial Virus [86]. In plants, eEF1 $\alpha$ has been shown to bind tRNA-like structures (TLSs) in the 3' region of Turnip yellow mosaic virus (TYMV), Tobacco mosaic virus (TMV), and Brome mosaic virus (BMV) [87,88]. The aminoacylation of the TLSs enhance virus protein translation and facilitates virus RNA encapsidation [89-91]. The interaction between the ER-localized P3 from the Soybean mosaic virus (SMV) and $e E F 1 \alpha$ has been shown to be essential for virulence in the susceptible host [82]. Furthermore, eEF1A from N. benthamiana has been identified as a pro-viral factor required for the Tomato spotted wilt virus disease (TSWV) [92]. Maize possesses at least eleven eEFs, from which nine are annotated in the MaizeGDB database as "elongation factor $\alpha(\text { elf } \alpha)^{\prime \prime}$ (Supplementary Table 1). A variety of functional transcripts are associated to each of them; for example, elfa1 has 15 transcripts associated with this gene model. In our case, we speculate that high expression levels of elfa10 and elfa3 (Table 1, red) suggest these factors interact with SCMV in a yet unforeseen manner. In contrast, RL showed down-regulation in elfa12 and Zm-elfa (Table 1, blue). It is possible that elfa10 and elfa3 interact better with SCMV, maintaining low expression of the non-interactors elfa12 and Zm-elfa. Additionally, according to the data obtained in the expression pattern cluster profiles, different transcripts of the same gene model with high or low basal levels could be found among the clusters, suggesting an intricate regulation of these factors in maize during SCMV infection. It will be necessary to dissect the interaction of elfa10, elfa3, elfa12 and $\mathrm{Zm}$-elfa with SCMV to disentangle the myriad of translation factors involved in this hostvirus interaction.

Besides the large number of possible important genes for resistance, SNPs also need to be considered for some of the candidate genes discussed here, adding more complexity than anticipated in maize-SCMV interactions. Finding them will contribute to breeding programs against devastating virus diseases in monocots.

\section{Materials and Methods}

4.1. Virus and Plant Matherials 
Sugarcane mosaic virus isolate Veracruz 1 (SCMV-VER1) was used as the viral inoculum. Biologically infected maize tissue collected from symptomatic plants in Veracruz fields was stored in an ultra-freezer at $-70{ }^{\circ} \mathrm{C}$. The inoculum was reactivated by inoculated B73 susceptible plants with a mix of approximately $500 \mathrm{mg}$ of ground infected tissue, $1 \mathrm{ml}$ of PBS 1X buffer, and a small amount of carborundum. Leaves showing classic mosaic symptoms were ground and used to further inoculate the available leaves of maize plants in the third true leaf stage.

Three maize lines were used in this work. The resistant CI-RL1 line, from CIMMYT, was propagated in a greenhouse, and seeds were stored in a cold room. The susceptible line B73 was donated from the CIMMYT seed bank. Additionally, a F1 line was generated in the winter field in Vallarta, using CI-RL1 as the female and B73 as the pollen donor.

Leaves from mock- or virus-inoculated plants were surveyed by RT-PCR, for the presence of the $\mathrm{CP}$ cistron in B73 and F1 plants, in order to confirm viral infection after RNA extraction and quantification in a mix of four biological replicas. Overall, 12 pools of total RNA samples were generated: 2 for B73, 2 for RL, and 2 for F1 virus inoculates, plus 2 for each line for mock-inoculated plants. mRNA was isolated from each of the pools using Dynabeads, following the manufacturer's protocol. The concentration of each pool was measured again and the RIN of each of the pools was obtained. Samples were then shipped for RNA-seq analysis.

\subsection{Experimental Design and Sequencing}

Six plants of each line were grown in a greenhouse until they reached the third true leaf stage. All of the available leaves were inoculated with a mix of $500 \mathrm{mg}$ of symptomatic ground tissue, 1X PBS buffer, and carborundum. Plants were observed daily and, at 17 $\mathrm{dpi}$, leaves were collected and stored in an ultra-freezer at $-70{ }^{\circ} \mathrm{C}$. Total RNA was extracted from $100 \mathrm{mg}$ of frozen tissue using TRIzol reagent (ThermoFisher, Waltham, MA, USA), according to the manufacturer's instructions. The concentration of the resulting total RNA was determined with Nanodrop 2000. Four biological replicates of each sample at the same concentration were mixed. mRNA was purified from the 12 total RNA pools using Dynabeads (Invitrogen, MA, USA), according to manufacturer's instructions. A total of 12 paired-end $(2 \times 150)$ RNA-Seq libraries (six treatments, two replicates per treatment) were prepared and sequenced at the Beijing Genomics Institute facility, using DNBSEQ ${ }^{\mathrm{TM}}$ technology (BGI, CHN). The raw data are publicly available at the National Center for Biotechnology Information (NCBI), under BioProject accession PRJNA784537.

\subsection{Mapping and Differential Expression Analysis}

Trimming adaptors and cleaning of low-quality reads were conducted as described previously [93]. Mapping and expression quantification were conducted with Kallisto, as described by [94]. Reads that failed to map to the reference B73 transcriptome retrieved from monocots PLAZA 4.5 (https://bioinformatics.psb.ugent.be/plaza/versions/plaza_v4_5_monocots) were de novo assembled with Trinity under standard parameters [95]. Non-mapped reads were re-mapped to the de novo assembled transcripts and quantified as described earlier. The differential expression analysis was performed with Sleuth, as reported previously [96], comparing each infected maize line with their mock. Genes with a p-value of $\leq 0.01$ were considered as differentially expressed genes (DEGs). Identification of expression pattern clusters (co-expressed transcript clusters) was carried out using the Clust software, under standard parameters with TPM normalization [97].

\subsection{Functional Enrichment}

Gene annotation data for reference B73 transcripts was downloaded from monocots PLAZA 4.5 (https://bioinformatics.psb.ugent.be/plaza/versions/plaza_v4_5_monocots). For the de novo assembled transcripts, annotation was carried out using the Trinotate 
pipeline [98]. Then, functional enrichment analysis was performed with AgriGOv2 with Fisher test considering a Yekutieli false discovery rate of $\leq 0.05$ [99]. Enrichment results were summarized with REVIGO, with a small similarity of 0.5 and SimRel as the semantic similarity score [100].

\subsection{Gene Expression Validation}

To validate the bioinformatic results of gene expression, we carried out RT-qPCR of 11 candidate genes (Figure Supplementary 4), using the same RNA from sequencing. cDNA were synthesized using the RevertAid minus H enzyme. RT-qPCR reactions were performed in an CFX96 Real-time system (BioRad, Hercules, CA, USA) with Maxima SYBR Green qPCR/ROX qPCR Master Mix (2X) (Thermo Scientific, Linhus, Lithuania). Relative expression was calculated using the $\mathrm{Ct}$ values and normalized with $\beta$-tubulin as the housekeeping gene.

\section{Conclusions}

We performed RNAseq analysis of a resistant line (RL), a susceptible line (B73), and the F1 progeny of both lines. . GO enrichment analysis of cellular components led to the identification of putative candidate genes which were up-regulated in RL, coding for an integral component of the cellular membrane. We chose the gene annotated as an ABC transporter as a likely candidate for resistance. We also found two genes implied in longdistance movement-ZmPiezo and ZmPVIP-in maize. These three genes were validated using qRT-PCR.

Transcript expression patterns provided evidence of the diverse (and, hence, complex) behavior of the eIF4E homologs in maize, particularly the $e E F 1 \alpha$ factors. Furthermore, two new maize gene models-ZmeIF4E and ZmeEF1 $\alpha$ - were identified as possibly being involved in the SCMV interaction.

More work is needed to understand how the genes identified in this work-ZmeIF4E, $\mathrm{Zm}$-elfa, ZmPiezo, ZmPVIP and $\mathrm{ABC}$ transporter-are involved in maize potyvirus resistance.

Supplementary Materials: The following are available online at www.mdpi.com/xxx/s1, Table S1: eIFs and eEFs annotated in the MaizeGDB database.; Table S2: List of the total of resistance candidate genes searched for in the expression pattern clusters.; Table S3: Candidate genes found in group 1 (blue) and group 2 (red) of the expression pattern clusters.; Figure S4: Validation of candidate genes by RT-qPCR.

Author Contributions: Conceptualization, G.R., P.V., and L.S.; methodology, G.R.; software, P.V.; validation, G.R.; formal analysis, P.V.; resources, L.S.; data curation, P.V.; writing-original draft preparation, G.R., P.V., and L.S.; writing - review and editing, G.R., P.V., and L.S.; visualization, G.R. and P.V.; supervision, L.S.; project administration, L.S.; funding acquisition, L.S. All authors have read and agreed to the published version of the manuscript.

Funding: G.R. and P.V. were supported by Consejo Nacional de Ciencia y Tecnologia postgraduate scholarships (CVU/Becario: 420714/262948 and 1024179/, respectively). This research was funded through PlanTecc and Cinvestav funds, in addition to personal funding by L.S.

\section{Data Availability Statement:}

Acknowledgments: The authors wish to thank Natzul Santoyo Villa for their technical help, as well as CYMMIT for donating the B73 seeds under the request number S2018_400684999 and S2020_623050816.

Conflicts of Interest: The authors declare no conflict of interest. The funders had no role in the design of the study; in the collection, analyses, or interpretation of data; in the writing of the manuscript, or in the decision to publish the results.

\section{References}


1. Redinbaugh, M.G.; Stewart, L.R. Maize Lethal Necrosis: An Emerging, Synergistic Viral Disease. Annu Rev Virol 2018, 5, 301322, doi:10.1146/annurev-virology-092917-043413.

2. Xie, L.; Zhang, J.; Wang, Q.; Meng, C.; Hong, J.; Zhou, X. Characterization of Maize Chlorotic Mottle Virus Associated with Maize Lethal Necrosis Disease in China. Journal of Phytopathology 2011, 159, 191-193, doi:10.1111/j.1439-0434.2010.01745.x.

3. Kiruwa, F.H.; Feyissa, T.; Ndakidemi*, P.A. Insights of Maize Lethal Necrotic Disease: A Major Constraint to Maize Production in East Africa. AJMR 2016, 10, 271-279, doi:10.5897/AJMR2015.7534.

4. Dangl, J.L.; Jones, J.D.G. Plant Pathogens and Integrated Defence Responses to Infection. Nature 2001, 411, 826-833, doi:10.1038/35081161.

5. Ausubel, F.M. Are Innate Immune Signaling Pathways in Plants and Animals Conserved? Nat Immunol 2005, 6, 973-979, doi:10.1038/ni1253.

6. Chisholm, S.T.; Coaker, G.; Day, B.; Staskawicz, B.J. Host-Microbe Interactions: Shaping the Evolution of the Plant Immune Response. Cell 2006, 124, 803-814, doi:10.1016/j.cell.2006.02.008.

7. Jones, J.D.G.; Dangl, J.L. The Plant Immune System. Nature 2006, 444, 323-329, doi:10.1038/nature05286.

8. Nurnberger, T.; Brunner, F.; Kemmerling, B.; Piater, L. Innate Immunity in Plants and Animals: Striking Similarities and Obvious Differences. Immunol Rev 2004, 198, 249-266, doi:10.1111/j.0105-2896.2004.0119.x.

9. Bigeard, J.; Colcombet, J.; Hirt, H. Signaling Mechanisms in Pattern-Triggered Immunity (PTI). Molecular Plant 2015, 8, 521-539, doi:10.1016/j.molp.2014.12.022.

10. DeYoung, B.J.; Innes, R.W. Plant NBS-LRR Proteins in Pathogen Sensing and Host Defense. Nat Immunol 2006, 7, 1243-1249, doi:10.1038/ni1410.

11. Boller, T.; Felix, G. A Renaissance of Elicitors: Perception of Microbe-Associated Molecular Patterns and Danger Signals by Pattern-Recognition Receptors. Annu. Rev. Plant Biol. 2009, 60, 379-406, doi:10.1146/annurev.arplant.57.032905.105346.

12. Liu, L.; Sonbol, F.-M.; Huot, B.; Gu, Y.; Withers, J.; Mwimba, M.; Yao, J.; He, S.Y.; Dong, X. Salicylic Acid Receptors Activate Jasmonic Acid Signalling through a Non-Canonical Pathway to Promote Effector-Triggered Immunity. Nat Commun 2016, 7 , 13099, doi:10.1038/ncomms13099.

13. Velásquez, A.C.; Castroverde, C.D.M.; He, S.Y. Plant-Pathogen Warfare under Changing Climate Conditions. Current Biology 2018, 28, R619-R634, doi:10.1016/j.cub.2018.03.054.

14. Saleem, M.; Fariduddin, Q.; Castroverde, C.D.M. Salicylic Acid: A Key Regulator of Redox Signalling and Plant Immunity. Plant Physiology and Biochemistry 2021, 168, 381-397, doi:10.1016/j.plaphy.2021.10.011.

15. Thomma, B.P.H.J.; Nürnberger, T.; Joosten, M.H.A.J. Of PAMPs and Effectors: The Blurred PTI-ETI Dichotomy. Plant Cell 2011, 23, 4-15, doi:10.1105/tpc.110.082602.

16. Nejat, N.; Mantri, N. Plant Immune System: Crosstalk Between Responses to Biotic and Abiotic Stresses the Missing Link in Understanding Plant Defence. Current Issues in Molecular Biology 2017, 1-16, doi:10.21775/cimb.023.001.

17. Wang, M.-B.; Masuta, C.; Smith, N.A.; Shimura, H. RNA Silencing and Plant Viral Diseases. MPMI 2012, 25, 1275-1285, doi:10.1094/MPMI-04-12-0093-CR.

18. Waterhouse, P.M.; Wang, M.-B.; Lough, T. Gene Silencing as an Adaptive Defence against Viruses. Nature 2001, 411, 834-842, doi:10.1038/35081168.

19. Mäkinen, K. Plant Susceptibility Genes as a Source for Potyvirus Resistance. Annals of Applied Biology 2020, 176, 122-129, doi:10.1111/aab.12562.

20. Bastet, A.; Robaglia, C.; Gallois, J.-L. EIF4E Resistance: Natural Variation Should Guide Gene Editing. Trends in Plant Science 2017, 22, 411-419, doi:10.1016/j.tplants.2017.01.008.

21. Dunoyer, P.; Thomas, C.; Harrison, S.; Revers, F.; Maule, A. A Cysteine-Rich Plant Protein Potentiates Potyvirus Movement through an Interaction with the Virus Genome-Linked Protein VPg. Journal of Virology 2004, 78, 2301-2309, doi:10.1128/JVI.78.5.2301-2309.2004.

22. Liu, Q.; Liu, H.; Gong, Y.; Tao, Y.; Jiang, L.; Zuo, W.; Yang, Q.; Ye, J.; Lai, J.; Wu, J.; et al. An Atypical Thioredoxin Imparts Early Resistance to Sugarcane Mosaic Virus in Maize. Molecular Plant 2017, 10, 483-497, doi:10.1016/j.molp.2017.02.002.

23. Vieira Dos Santos, C.; Rey, P. Plant Thioredoxins Are Key Actors in the Oxidative Stress Response. Trends in Plant Science 2006, 11, 329-334, doi:10.1016/j.tplants.2006.05.005.

24. Hisabori, T.; Motohashi, K.; Hosoya-Matsuda, N.; Ueoka-Nakanishi, H.; Romano, P.G.N. Towards a Functional Dissection of Thioredoxin Networks in Plant Cells. Photochem Photobiol 2006, 2006-02-27-IR-816, doi:10.1562/2006-02-27-IR-816.

25. Kang, B.-C.; Yeam, I.; Jahn, M.M. Genetics of Plant Virus Resistance. Annu. Rev. Phytopathol. 2005, 43, 581-621, doi:10.1146/annurev.phyto.43.011205.141140.

26. Garcia-Ruiz, H. Susceptibility Genes to Plant Viruses. Viruses 2018, 10, 484, doi:10.3390/v10090484.

27. Lellis, A.D.; Kasschau, K.D.; Whitham, S.A.; Carrington, J.C. Loss-of-Susceptibility Mutants of Arabidopsis Thaliana Reveal an Essential Role for EIF(Iso)4E during Potyvirus Infection. Current Biology 2002, 12, 1046-1051, doi:10.1016/S0960-9822(02)008989.

28. Lei, J.D. Mechanisms of Resistance in Corn to Maize Dwarf Mosaic Virus. Phytopathology 1986, 76, 1034, doi:10.1094/Phyto-761034.

29. Chaves-Bedoya, G.; Espejel, F.; Alcalá-Briseño, R.I.; Hernández-Vela, J.; Silva-Rosales, L. Short Distance Movement of Genomic Negative Strands in a Host and Nonhost for Sugarcane Mosaic Virus (SCMV). Virology Journal 2011, 8, 15, doi:10.1186/1743422X-8-15. 
30. Nagy, P.D.; Pogany, J. The Dependence of Viral RNA Replication on Co-Opted Host Factors. Nat Rev Microbiol 2012, 10, 137149, doi:10.1038/nrmicro2692.

31. Mäkinen, K.; Hafren, A. Intracellular Coordination of Potyviral RNA Functions in Infection. Frontiers in Plant Science 2014, 5, 110, doi:10.3389/fpls.2014.00110.

32. Reichel, C.; Beachy, R.N. Tobacco Mosaic Virus Infection Induces Severe Morphological Changes of the Endoplasmic Reticulum. PNAS 1998, 95, 11169-11174, doi:10.1073/pnas.95.19.11169.

33. Wileman, T. Aggresomes and Autophagy Generate Sites for Virus Replication. Science 2006, 312, 875-878, doi:10.1126/science.1126766.

34. Wei, T.; Huang, T.-S.; McNeil, J.; Laliberté, J.-F.; Hong, J.; Nelson, R.S.; Wang, A. Sequential Recruitment of the Endoplasmic Reticulum and Chloroplasts for Plant Potyvirus Replication. Journal of Virology 2010, 84, 799-809, doi:10.1128/JVI.01824-09.

35. Schaad, M.C.; Jensen, P.E.; Carrington, J.C. Formation of Plant RNA Virus Replication Complexes on Membranes: Role of an Endoplasmic Reticulum-Targeted Viral Protein. The EMBO Journal 1997, 16, 4049-4059, doi:10.1093/emboj/16.13.4049.

36. Kräutler, B. Breakdown of Chlorophyll in Higher Plants-Phyllobilins as Abundant, Yet Hardly Visible Signs of Ripening, Senescence, and Cell Death. Angewandte Chemie International Edition 2016, 55, 4882-4907, doi:10.1002/anie.201508928.

37. Hörtensteiner, S. Chlorophyll Degradation During Senescence. Annual Review of Plant Biology 2006, 57, 55-77, doi:10.1146/annurev.arplant.57.032905.105212.

38. Tamary, E.; Nevo, R.; Naveh, L.; Levin-Zaidman, S.; Kiss, V.; Savidor, A.; Levin, Y.; Eyal, Y.; Reich, Z.; Adam, Z. Chlorophyll Catabolism Precedes Changes in Chloroplast Structure and Proteome during Leaf Senescence. Plant Direct 2019, 3, e00127, doi:10.1002/pld3.127.

39. Bianchi, M.E. DAMPs, PAMPs and Alarmins: All We Need to Know about Danger. Journal of Leukocyte Biology 2007, 81, 1-5, doi:10.1189/jlb.0306164.

40. Choi, H.W.; Klessig, D.F. DAMPs, MAMPs, and NAMPs in Plant Innate Immunity. BMC Plant Biol 2016, 16, 232, doi:10.1186/s12870-016-0921-2.

41. Tang, D.; Kang, R.; Coyne, C.B.; Zeh, H.J.; Lotze, M.T. PAMPs and DAMPs: Signal 0s That Spur Autophagy and Immunity. Immunological Reviews 2012, 249, 158-175, doi:10.1111/j.1600-065X.2012.01146.x.

42. Kadota, Y.; Sklenar, J.; Derbyshire, P.; Stransfeld, L.; Asai, S.; Ntoukakis, V.; Jones, J.D.; Shirasu, K.; Menke, F.; Jones, A.; et al. Direct Regulation of the NADPH Oxidase RBOHD by the PRR-Associated Kinase BIK1 during Plant Immunity. Molecular Cell 2014, 54, 43-55, doi:10.1016/j.molcel.2014.02.021.

43. Apel, K.; Hirt, H. REACTIVE OXYGEN SPECIES: Metabolism, Oxidative Stress, and Signal Transduction. Annual Review of Plant Biology 2004, 55, 373-399, doi:10.1146/annurev.arplant.55.031903.141701.

44. Demidchik, V. Mechanisms of Oxidative Stress in Plants: From Classical Chemistry to Cell Biology. Environmental and Experimental Botany 2015, 109, 212-228, doi:10.1016/j.envexpbot.2014.06.021.

45. Ellis, J. Proteins as Molecular Chaperones. Nature 1987, 328, 378-379, doi:10.1038/328378a0.

46. Tyedmers, J.; Mogk, A.; Bukau, B. Cellular Strategies for Controlling Protein Aggregation. Nat Rev Mol Cell Biol 2010, 11, 777788, doi:10.1038/nrm2993.

47. Bukau, B.; Weissman, J.; Horwich, A. Molecular Chaperones and Protein Quality Control. Cell 2006, 125, 443-451, doi:10.1016/j.cell.2006.04.014.

48. Hafrén, A.; Hofius, D.; Rönnholm, G.; Sonnewald, U.; MÄkinen, K. HSP70 and Its Cochaperone CPIP Promote Potyvirus Infection in Nicotiana Benthamiana by Regulating Viral Coat Protein Functions. The Plant Cell 2010, 22, 523-535, doi:10.1105/tpc.109.072413.

49. Verchot, J. Cellular Chaperones and Folding Enzymes Are Vital Contributors to Membrane Bound Replication and Movement Complexes during Plant RNA Virus Infection. Frontiers in Plant Science 2012, 3, 275, doi:10.3389/fpls.2012.00275.

50. Lubkowska, A.; Pluta, W.; Strońska, A.; Lalko, A. Role of Heat Shock Proteins (HSP70 and HSP90) in Viral Infection. IJMS 2021, 22, 9366, doi:10.3390/ijms22179366.

51. Gorovits, R.; Czosnek, H. The Involvement of Heat Shock Proteins in the Establishment of Tomato Yellow Leaf Curl Virus Infection. Front. Plant Sci. 2017, 8, doi:10.3389/fpls.2017.00355.

52. Sullivan, C.S.; Pipas, J.M. The Virus-Chaperone Connection. Virology 2001, 287, 1-8, doi:10.1006/viro.2001.1038.

53. Higgins, C.F. ABC Transporters: From Microorganisms to Man. Annu Rev Cell Biol 1992, 8, 67-113, doi:10.1146/annurev.cb.08.110192.000435.

54. Moons, A. Ospdr9, Which Encodes a PDR-Type ABC Transporter, Is Induced by Heavy Metals, Hypoxic Stress and Redox Perturbations in Rice Roots11The Nucleotide Sequence Reported in This Paper Has Been Submitted to the EMBL, GenBank and DDBJ Nucleotide Sequence Databases with the Accession Number AY271618. FEBS Letters 2003, 553, 370-376, doi:10.1016/S0014-5793(03)01060-3.

55. Stukkens, Y.; Bultreys, A.; Grec, S.; Trombik, T.; Vanham, D.; Boutry, M. NpPDR1, a Pleiotropic Drug Resistance-Type ATPBinding Cassette Transporter from Nicotiana Plumbaginifolia, Plays a Major Role in Plant Pathogen Defense. Plant Physiol 2005, 139, 341-352, doi:10.1104/pp.105.062372.

56. Sun, D.; Zhang, X.; Li, S.; Jiang, C.-Z.; Zhang, Y.; Niu, L. LrABCF1, a GCN-Type ATP-Binding Cassette Transporter from Lilium Regale, Is Involved in Defense Responses against Viral and Fungal Pathogens. Planta 2016, 244, 1185-1199, doi:10.1007/s00425016-2576-5. 
57. Ellis, J.G.; Lagudah, E.S.; Spielmeyer, W.; Dodds, P.N. The Past, Present and Future of Breeding Rust Resistant Wheat. Frontiers in Plant Science 2014, 5, 641, doi:10.3389/fpls.2014.00641.

58. Krattinger, S.G.; Kang, J.; Bräunlich, S.; Boni, R.; Chauhan, H.; Selter, L.L.; Robinson, M.D.; Schmid, M.W.; Wiederhold, E.; Hensel, G.; et al. Abscisic Acid Is a Substrate of the ABC Transporter Encoded by the Durable Wheat Disease Resistance Gene Lr34. New Phytologist 2019, 223, 853-866, doi:10.1111/nph.15815.

59. Sucher, J.; Boni, R.; Yang, P.; Rogowsky, P.; Büchner, H.; Kastner, C.; Kumlehn, J.; Krattinger, S.G.; Keller, B. The Durable Wheat Disease Resistance Gene Lr34 Confers Common Rust and Northern Corn Leaf Blight Resistance in Maize. Plant Biotechnology Journal 2017, 15, 489-496, doi:10.1111/pbi.12647.

60. Dufresne, P.J.; Ubalijoro, E.; Fortin, M.G.; Laliberté, J.-F. Arabidopsis Thaliana Class II Poly(A)-Binding Proteins Are Required for Efficient Multiplication of Turnip Mosaic Virus. Journal of General Virology 2008, 89, 2339-2348, doi:10.1099/vir.0.2008/0021390 .

61. Zhang, L.; Chen, H.; Brandizzi, F.; Verchot, J.; Wang, A. The UPR Branch IRE1-BZIP60 in Plants Plays an Essential Role in Viral Infection and Is Complementary to the Only UPR Pathway in Yeast. PLoS Genet 2015, 11, e1005164, doi:10.1371/journal.pgen.1005164.

62. Reagan, B.C.; Burch-Smith, T.M. Viruses Reveal the Secrets of Plasmodesmal Cell Biology. MPMI 2020, 33, 26-39, doi:10.1094/MPMI-07-19-0212-FI.

63. Anjanappa, R.B.; Mehta, D.; Okoniewski, M.J.; Szabelska-Beręsewicz, A.; Gruissem, W.; Vanderschuren, H. Molecular Insights into Cassava Brown Streak Virus Susceptibility and Resistance by Profiling of the Early Host Response. Molecular Plant Pathology 2018, 19, 476-489, doi:10.1111/mpp.12565.

64. Kauss, H. Callose Biosynthesis as a Ca2+-Regulated Process and Possible Relations to the Induction of Other Metabolic Changes. Journal of Cell Science 1985, 1985, 89-103, doi:10.1242/jcs.1985.Supplement_2.5.

65. Zavaliev, R.; Ueki, S.; Epel, B.L.; Citovsky, V. Biology of Callose ( $\beta-1,3-G l u c a n)$ Turnover at Plasmodesmata. Protoplasma 2011, 248, 117-130, doi:10.1007/s00709-010-0247-0.

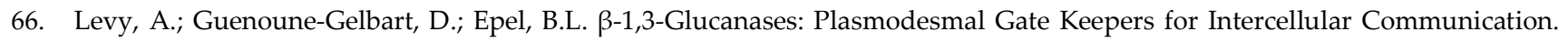
Plant Signaling \& Behavior 2007, 2, 404-407, doi:10.4161/psb.2.5.4334.

67. Pastor, V.; Luna, E.; Ton, J.; Cerezo, M.; García-Agustín, P.; Flors, V. Fine Tuning of Reactive Oxygen Species Homeostasis Regulates Primed Immune Responses in Arabidopsis. MPMI 2013, 26, 1334-1344, doi:10.1094/MPMI-04-13-0117-R.

68. Beris, D.; Kotsaridis, K.; Vakirlis, N.; Termentzi, A.; Theologidis, I.; Moury, B.; Vassilakos, N. The Plasma Membrane Cation Binding Protein 1 Affects Accumulation of Potato Virus Y in Pepper Both at the Systemic Level and in Protoplasts. Virus Research 2020, 280, 197899, doi:10.1016/j.virusres.2020.197899.

69. Vijayapalani, P.; Maeshima, M.; Nagasaki-Takekuchi, N.; Miller, W.A. Interaction of the Trans-Frame Potyvirus Protein P3NPIPO with Host Protein PCaP1 Facilitates Potyvirus Movement. PLoS Pathog 2012, 8, e1002639, doi:10.1371/journal.ppat.1002639.

70. Harries, P.A.; Park, J.-W.; Sasaki, N.; Ballard, K.D.; Maule, A.J.; Nelson, R.S. Differing Requirements for Actin and Myosin by Plant Viruses for Sustained Intercellular Movement. Proceedings of the National Academy of Sciences 2009, 106, 17594-17599, doi:10.1073/pnas.0909239106.

71. Amari, K.; Boutant, E.; Hofmann, C.; Schmitt-Keichinger, C.; Fernandez-Calvino, L.; Didier, P.; Lerich, A.; Mutterer, J.; Thomas, C.L.; Heinlein, M.; et al. A Family of Plasmodesmal Proteins with Receptor-Like Properties for Plant Viral Movement Proteins. PLoS Pathog 2010, 6, e1001119, doi:10.1371/journal.ppat.1001119.

72. Thomas, C.L.; Bayer, E.M.; Ritzenthaler, C.; Fernandez-Calvino, L.; Maule, A.J. Specific Targeting of a Plasmodesmal Protein Affecting Cell-to-Cell Communication. PLoS Biol 2008, 6, e7, doi:10.1371/journal.pbio.0060007.

73. Ridone, P.; Vassalli, M.; Martinac, B. Piezo1 Mechanosensitive Channels: What Are They and Why Are They Important. Biophys Rev 2019, 11, 795-805, doi:10.1007/s12551-019-00584-5.

74. Zhang, Z.; Tong, X.; Liu, S.-Y.; Chai, L.-X.; Zhu, F.-F.; Zhang, X.-P.; Zou, J.-Z.; Wang, X.-B. Genetic Analysis of a Piezo-like Protein Suppressing Systemic Movement of Plant Viruses in Arabidopsis Thaliana. Sci Rep 2019, 9, 3187, doi:10.1038/s41598019-39436-3.

75. Gutierrez Sanchez, P.A.; Babujee, L.; Jaramillo Mesa, H.; Arcibal, E.; Gannon, M.; Halterman, D.; Jahn, M.; Jiang, J.; Rakotondrafara, A.M. Overexpression of a Modified EIF4E Regulates Potato Virus Y Resistance at the Transcriptional Level in Potato. BMC Genomics 2020, 21, 18, doi:10.1186/s12864-019-6423-5.

76. Ivanov, K.I.; Eskelin, K.; Lõhmus, A.; Mäkinen, K.Y. 2014 Molecular and Cellular Mechanisms Underlying Potyvirus Infection. Journal of General Virology 95, 1415-1429, doi:10.1099/vir.0.064220-0.

77. Spriggs, K.A.; Stoneley, M.; Bushell, M.; Willis, A.E. Re-Programming of Translation Following Cell Stress Allows IRES-Mediated Translation to Predominate. Biology of the Cell 2008, 100, 27-38, doi:10.1042/BC20070098.

78. Sheikh, M.S.; Fornace, A.J. Regulation of Translation Initiation Following Stress. Oncogene 1999, 18, 6121-6128, doi:10.1038/sj.onc.1203131.

79. Contreras-Paredes, C.A.; Silva-Rosales, L.; Daròs, J.-A.; Alejandri-Ramírez, N.D.; Dinkova, T.D. The Absence of Eukaryotic Initiation Factor EIF(Iso)4E Affects the Systemic Spread of a Tobacco Etch Virus Isolate in Arabidopsis Thaliana. MPMI 2013, 26, 461470, doi:10.1094/MPMI-09-12-0225-R.

80. Cristiano, L. The Pseudogenes of Eukaryotic Translation Elongation Factors (EEFs): Role in Cancer and Other Human Diseases. Genes \& Diseases 2021, doi:10.1016/j.gendis.2021.03.009. 
81. Mateyak, M.K.; Kinzy, T.G. EEF1A: Thinking Outside the Ribosome. J Biol Chem 2010, 285, 21209-21213, doi:10.1074/jbc.R110.113795.

82. Luan, H.; Shine, M.B.; Cui, X.; Chen, X.; Ma, N.; Kachroo, P.; Zhi, H.; Kachroo, A. The Potyviral P3 Protein Targets Eukaryotic Elongation Factor 1A to Promote the Unfolded Protein Response and Viral Pathogenesis. Plant Physiology 2016, 172, 221-234, doi:10.1104/pp.16.00505.

83. Sasikumar, A.N.; Perez, W.B.; Kinzy, T.G. The Many Roles of the Eukaryotic Elongation Factor 1 Complex. WIREs RNA 2012, 3 , 543-555, doi:10.1002/wrna.1118.

84. Cimarelli, A.; Luban, J. Translation Elongation Factor 1-Alpha Interacts Specifically with the Human Immunodeficiency Virus Type 1 Gag Polyprotein. Journal of Virology 1999, 73, 5388-5401, doi:10.1128/JVI.73.7.5388-5401.1999.

85. Blackwell, J.L.; Brinton, M.A. Translation Elongation Factor-1 Alpha Interacts with the 3' Stem-Loop Region of West Nile Virus Genomic RNA. J Virol 1997, 71, 6433-6444, doi:10.1128/jvi.71.9.6433-6444.1997.

86. Wei, T.; Li, D.; Marcial, D.; Khan, M.; Lin, M.-H.; Snape, N.; Ghildyal, R.; Harrich, D.; Spann, K. The Eukaryotic Elongation Factor 1A Is Critical for Genome Replication of the Paramyxovirus Respiratory Syncytial Virus. PLoS ONE 2014, 9, e114447, doi:10.1371/journal.pone.0114447.

87. Dreher, T.W. Role of TRNA-like Structures in Controlling Plant Virus Replication. Virus Research 2009, 139, 217-229, doi:10.1016/j.virusres.2008.06.010.

88. Li, D.; Wei, T.; Abbott, C.M.; Harrich, D. The Unexpected Roles of Eukaryotic Translation Elongation Factors in RNA Virus Replication and Pathogenesis. Microbiology and Molecular Biology Reviews 2013, 77, 253-266, doi:10.1128/MMBR.00059-12.

89. Matsuda, D.; Dreher, T.W. The TRNA-like Structure of Turnip Yellow Mosaic Virus RNA Is a 3'-Translational Enhancer. Virology 2004, 321, 36-46, doi:10.1016/j.virol.2003.10.023.

90. Choi, Y.G.; Dreher, T.W.; Rao, A.L.N. TRNA Elements Mediate the Assembly of an Icosahedral RNA Virus. PNAS 2002, 99, 655660, doi:10.1073/pnas.022618199.

91. Annamalai, P.; Rao, A.L.N. In Vivo Packaging of Brome Mosaic Virus RNA3, but Not RNAs 1 and 2, Is Dependent on a CisActing 3' TRNA-Like Structure. Journal of Virology 2007, 81, 173-181, doi:10.1128/JVI.01500-06.

92. Helderman, T.A.; Deurhof, L.; Bertran, A.; Boeren, S.; Fokkens, L.; Kormelink, R.; Joosten, M.H.A.J.; Prins, M.; van den Burg, H.A. An Isoform of the Eukaryotic Translation Elongation Factor 1A (EEF1a) Acts as a Pro-Viral Factor Required for Tomato Spotted Wilt Virus Disease in Nicotiana Benthamiana. Viruses 2021, 13, 2190, doi:10.3390/v13112190.

93. Fabbro, C.D.; Scalabrin, S.; Morgante, M.; Giorgi, F.M. An Extensive Evaluation of Read Trimming Effects on Illumina NGS Data Analysis. PLOS ONE 2013, 8, e85024, doi:10.1371/journal.pone.0085024.

94. Vargas-Mejía, P.; Vega-Arreguín, J.; Chávez-Calvillo, G.; Ibarra-Laclette, E.; Silva-Rosales, L. Differential Accumulation of Innate- and Adaptive-Immune-Response-Derived Transcripts during Antagonism between Papaya Ringspot Virus and Papaya Mosaic Virus. Viruses 2020, 12, 230, doi:10.3390/v12020230.

95. Haas, B.J.; Papanicolaou, A.; Yassour, M.; Grabherr, M.; Blood, P.D.; Bowden, J.; Couger, M.B.; Eccles, D.; Li, B.; Lieber, M.; et al. De Novo Transcript Sequence Reconstruction from RNA-Seq: Reference Generation and Analysis with Trinity. Nat Protoc 2013, 8, doi:10.1038/nprot.2013.084.

96. Yi, L.; Pimentel, H.; Bray, N.L.; Pachter, L. Gene-Level Differential Analysis at Transcript-Level Resolution. Genome Biology 2018, 19, 53, doi:10.1186/s13059-018-1419-z.

97. Abu-Jamous, B.; Kelly, S. Clust: Automatic Extraction of Optimal Co-Expressed Gene Clusters from Gene Expression Data. Genome Biology 2018, 19, 172, doi:10.1186/s13059-018-1536-8.

98. Bryant, D.M.; Johnson, K.; DiTommaso, T.; Tickle, T.; Couger, M.B.; Payzin-Dogru, D.; Lee, T.J.; Leigh, N.D.; Kuo, T.-H.; Davis, F.G.; et al. A Tissue-Mapped Axolotl De Novo Transcriptome Enables Identification of Limb Regeneration Factors. Cell Reports 2017, 18, 762-776, doi:10.1016/j.celrep.2016.12.063.

99. Tian, T.; Liu, Y.; Yan, H.; You, Q.; Yi, X.; Du, Z.; Xu, W.; Su, Z. AgriGO v2.0: A GO Analysis Toolkit for the Agricultural Community, 2017 Update. Nucleic Acids Res 2017, 45, W122-W129, doi:10.1093/nar/gkx382.

100. Supek, F.; Bošnjak, M.; Škunca, N.; Šmuc, T. REVIGO Summarizes and Visualizes Long Lists of Gene Ontology Terms. PLOS ONE 2011, 6, e21800, doi:10.1371/journal.pone.0021800. 